\title{
Vitamin D deficiency in African Americans is associated with a high risk of severe disease and mortality by SARS-CoV-2
}

\author{
Virna Margarita Martín Giménez ${ }^{1}$ Felipe Inserra ${ }^{2} \cdot$ León Ferder $^{2} \cdot$ Joxel García $^{3} \cdot$ Walter Manucha $\mathbb{E}^{4}$
}

Received: 5 May 2020 / Revised: 28 July 2020 / Accepted: 5 August 2020 / Published online: 13 August 2020

(c) Springer Nature Limited 2020

\section{To the Editor:}

COVID-19 infection results in an elevated risk of pulmonary complications and mortality in the hypertensive, diabetic, and old age individuals and patients with cardiovascular or pulmonary diseases. This situation is critical in the African American (AA) population. As a paradigmatic representation of the state is of interest, mentioning that the morbidity and mortality rates by COVID-19 in AA are the highest among many other populations, as well as the mortality rate is 6-fold higher compared with white people [1].

There are many health disparities in AA like high incidence rates of obesity, diabetes, high blood pressure, cardiovascular and renal diseases, among others. The usual explanation for these differences is the low socioeconomic status and educational levels, the social environment, lifestyle habits, and less access to health care services. However, there are pieces of evidence that these non-favorable conditions are not enough, and there are other influential factors that may help to a better approach to the real problem, like some genetic polymorphism and epigenetic-driven changes [2]. In this sense, of medical relevance are the differences in renin-angiotensin-aldosterone systems (RAAS), renal sodium manages [3], and -of interest for this letter-, the low levels of serum vitamin D [4, 5] (Fig. 1).

$\triangle$ Walter Manucha

wmanucha@yahoo.com.ar

1 Instituto de Investigaciones en Ciencias Químicas, Facultad de Ciencias Químicas y Tecnológicas, Universidad Católica de Cuyo, San Juan, Argentina

2 Universidad Maimónides, Buenos Aires, Argentina

3 AMBITNA, Ambitious Solutions for Health Cures, Chevy Chase, MD, USA

4 Instituto de Medicina y Biología Experimental de Cuyo (IMBECU, CCT-Mendoza, CONICET) y Facultad de Ciencias Médicas, Universidad Nacional de Cuyo, Mendoza, Argentina
AA people have a high genetic ability to retain salt [6]. The sodium retention observed in black people causes the inhibition of systemic RAAS (sRAAS) by negative feedback. The high prevalence of high blood pressure levels in this population is a clinical manifestation of these disarrangements. Also, high blood pressure would be one of the main risk factors for SARs-CoV-2 infection [7], and RAAS would be an essential part of the pulmonary tissue injury [8].

AA people use drugs that blockade RAAS despite its lower reduction in blood pressure compared whit its use in non AA patients, because of its protective cardiovascular effects. A recent publication had proposed the hypothesis that RAAS blocker drugs, could act as a risk factor for patients with SARS-CoV-2 infection, by increasing synthesis of angiotensin-converting enzyme 2 (ACE2) [9]. Indeed, there are experimental works that have demonstrated that the use of these drugs increases ACE2 levels [10], being the ACE2 receptor used by SARS-CoV-2 to enter and injury the cells [11]. An immediate reaction from the medical community and scientific societies, together with the appearance of the results of several studies, confirmed that blocking the RAAS was not harmful and could even be beneficial for the evolution of infected patients. After entering the cells, SARS-CoV-2 reduces protective ACE2 activity and function inducing, in turn, damage to pulmonary parenchyma. At the same time, the consequent imbalance of ACE/ACE2 enhances the harmful action of angiotensin II on the lungs of the infected patients [12].

Complementary, there is an association between high serum vitamin D levels and benefits on many aspects of health, including viral infection. Most of the AA people lack normal serum levels of vitamin $\mathrm{D}$, and the average of their serum levels is considerably lower than other populations [5] (Fig. 1).

Additionally, low serum vitamin D levels are associated with a higher number and severity of respiratory infections than people with normal levels [13]. Clinical trials have shown that vitamin $\mathrm{D}$ administration reduces respiratory 
Fig. 1 Imbalanced protective and harmful factors facing to COVID-19 infection in African Americans. Multiple genetic and epigenetic factors added to lack of the equilibrium between ACE/ACE2 are critical in the African American population since they predispose to get several diseases such as hypertension and diabetes mellitus which worsen the pathophysiology of COVID-19 infection and increase the morbidity and mortality in black people.

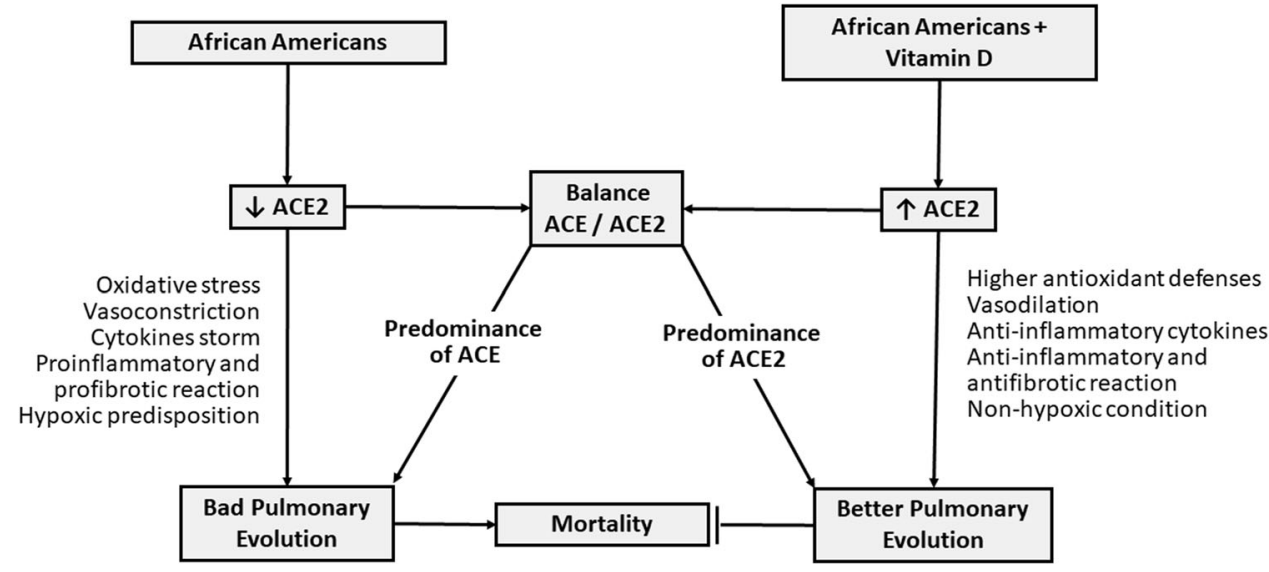

infections in healthy people, as well as in patients with chronic respiratory diseases, including cases with viral infection by COVID-19 [14]. It is known that vitamin D exerts this protective effect on respiratory tract mainly through three mechanisms: the preservation of tight junctions to avoid the immune cells infiltration of into the lungs and other respiratory organs, the destruction of enveloped viruses by the stimulation of cathelicidin and defensins, and the decrease in pro-inflammatory cytokines synthesis by the immune system modulation [15]. Moreover, vitamin $\mathrm{D}$ has been suggested as a natural antioxidant and antiinflammatory able to enhance the prognosis of lung pathologies [16]. Additionally, the combined actions of vitamin D and other endogenous molecules with strong antioxidant properties such as melatonin may provide a synergistic effect against COVID-19 infection and its lethal consequences [17].

To highlights, RAAS and vitamin D share its evolutionary origin, the ubiquity in many cells, and tissues such as the lungs. However, our group described an inverse relationship in both healthy in the disease conditions [18].

Some studies show that AA people present an imbalance between ACE/ACE2 as a consequence of overactivity of the RAAS pressor arm (ACE/Ang II/AT1 receptor) and lower activity in the RAAS depressor axis (ACE2/Ang(1-7)/Mas receptor), reducing ACE2 activity. The level of ACE2 in different tissues, especially in the lungs- seems to be crucial for the susceptibility, development, and progression for hypertension, cardiovascular risk, and pulmonary viral diseases as SARS-CoV-2 infection. There is experimental evidence that SARS-CoV-2 uses ACE2 receptors and the serine protease TMPRSS2 to enter the pulmonary cells trapping and downregulates ACE2 receptors reducing its activity [11]. The preservation of the integrity and functionality of the ACE2 receptor is associated and seems to be essential against viral infections and the maintenance of an adequate lung function. RAAS inhibition and vitamin D supplementation increase ACE2 levels and restore the ACE/ACE2 balance. Multiple studies showed that vitamin D antagonizes RAAS effects, especially by the reduction in the inflammatory response [19]. Furthermore, basic, epidemiological, and clinical research reinforces the idea that vitamin $\mathrm{D}$ protects from severe viral infections. Despite the lack of studies to define the adequate level of vitamin D to protect against viral infection, we agree with Grant et al., and estimate that a range between 40 and $60 \mathrm{mg} / \mathrm{dL}$ and the recommended dose to achieve this, between 5000 and 10,000 IU/day for several weeks [14].

Consequently, as is summarized in figure, our letter aims to generate discussion addressing plausible use of high doses of vitamin $\mathrm{D}$ in the AA population as a protective strategy in COVID-19 against both virus entrance, inflammatory storm, and inclusive, the death. As was proposed for other high-risk populations, and currently are ongoing at list ten randomized controlled trials [20], it is necessary to know whether vitamin D supplementation could be useful in the prevention and treatment of COVID-19 in the AA population.

Funding This work was supported by grants from the Research and Technology Council of Cuyo University (SECyT), Mendoza, Argentina, and from ANPCyT FONCyT, both of which were awarded to WM. Grant nos. PICT 2016-4541 and IP-COVID-19-931.

\section{Compliance with ethical standards}

Conflict of interest The authors declare that they have no conflict of interest.

Publisher's note Springer Nature remains neutral with regard to jurisdictional claims in published maps and institutional affiliations.

\section{References}

1. Yancy CW. COVID-19 and African Americans. JAMA. 2020. https://doi.org/10.1001/jama.2020.6548. 
2. Holmes L Jr. Enwere M, Williams J, Ogundele B, Chavan P, Piccoli T, et al. Black-white risk differentials in COVID-19 (SARSCOV2) transmission, mortality and case fatality in the United States: translational epidemiologic perspective and challenges. J Environ Res Public Health. 2020;17:4322.

3. Zilbermint M, Hannah-Shmouni F, Stratakis CA. Genetics of hypertension in African Americans and others of African descent. Int J Mol Sci. 2019;20:1081.

4. Liu X, Baylin A, Levy PD. Vitamin D deficiency and insufficiency among US adults: prevalence, predictors and clinical implications. Br J Nutr. 2018;119:928-36.

5. Orces C, Lorenzo C, Guarneros JE. The prevalence and determinants of vitamin D inadequacy among U.S. older adults: national health and nutrition examination survey 2007-14. Cureus. 2019;11:e5300. https://doi.org/10.7759/cureus.5300.

6. Lackland DT. Racial differences in hypertension: implications for high blood pressure management. Am J Med Sci. 2014;348:135-8.

7. Zuin M, Rigatelli G, Zuliani G, Rigatelli A, Mazza A, Roncon L Arterial hypertension and risk of death in patients with COVID-19 infection: systematic review and meta-analysis. J Infect. 2020. https://doi.org/10.1016/j.jinf.2020.03.059.

8. Busse LW, Chow JH, McCurdy MT, Khanna AK. COVID-19 and the RAAS-a potential role for angiotensin II? Crit Care. 2020;24:136.

9. Sommerstein R, Grani C. Rapid response: re: preventing a covid19 pandemic: ACE inhibitors as a potential risk factor for fatal Covid-19. BMJ. 2020;368:m810.

10. Li XC, Zhang J, Zhuo JL. The vasoprotective axes of the reninangiotensin system: physiological relevance and therapeutic implications in cardiovascular, hypertensive and kidney diseases. Pharm Res. 2017;125:21-38.

11. Hoffmann M, Kleine-Weber H, Schroeder S, Kruger N, Herrler T, Erichsen S, et al. SARS-CoV-2 cell entry depends on ACE2 and TMPRSS 2 and is blocked by a clinically proven protease inhibitor. Cell. 2020;181:1-10.
12. Kuba K, Imai Y, Rao S, Huan Y, Guo F, Guan B, et al. Angiotensin-converting enzyme 2 protects from severe acute lung failure. Nature. 2005;436:112-6.

13. Zhou YF, Luo BA, Qin LL. The association between vitamin D deficiency and community-acquired pneumonia: a meta-analysis of observational studies. Medicine. 2019;98:e17252. https://doi. org/10.1097/MD.0000000000017252.

14. Grant WB, Lahore H, McDonnell SL, Baggerly CA, French CB, Aliano JL, et al. Evidence that Vitamin D Supplementation Could Reduce Risk of Influenza and COVID-19 Infections and Deaths. Nutrients. 2020;12:E988. https://doi.org/10.3390/nu12040988.

15. Grant WB, Lahore H, McDonnell SL, Baggerly CA, French CB, Aliano JL, et al. Vitamin D supplementation could prevent and treat influenza, coronavirus, and pneumonia infections. Preprints. 2020; 2020030235. https://doi.org/10.20944/preprints202003.0235.v1.

16. Biswas S, Hwang JW, Kirkham PA, Rahman I. Pharmacological and dietary antioxidant therapies for chronic obstructive pulmonary disease. Curr Med Chem. 2013;20:1496-530.

17. Martín Giménez VM, Inserra F, Tajer CD, Mariani J, Ferder L, Reiter RJ, et al. Lungs as target of COVID-19 infection: protective common molecular mechanisms of vitamin $\mathrm{D}$ and melatonin as a new potential synergistic treatment. Life Sci. 2020;254:117808. https://doi.org/10.1016/j.lfs.2020.11780817.

18. Ferder M, Inserra F, Manucha W, Ferder L. The world pandemic of vitamin D deficiency could possibly be explained by cellular inflammatory response activity induced by the renin-angiotensin system. Am J Physiol Cell Physiol. 2013;304:C1027-39.

19. Xu J, Yang J, Chen J, Luo Q, Zhang Q, Zhang H. Vitamin D alleviates lipopolysaccharide-induced acute lung injury via regulation of the renin-angiotensin system. Mol Med Rep. 2017;16:7432-8.

20. Quesada-Gomez JM, Entrenas Castillo M, Bouillon R. Vitamin D receptor stimulation to reduce acute respiratory distress syndrome (ARDS) in patients with coronavirus SARS-CoV-2 infections. Steroid Biochem Mol Biol. 2020;105719. https://doi.org/10.1016/ j.jsbmb.2020.105719. 